\title{
Neoadjuvant Imatinib Therapy Followed by Surgery in a Rare Case of Rectal Gastrointestinal Stromal Tumor
}

\author{
Ashok Kumar ${ }^{1}$, Nalinikanta Ghosh ${ }^{2}$, Ashok Kumar ${ }^{3}$, Nishant Malviya ${ }^{4}$
}

\begin{abstract}
In patients with very low rectal tumors, intersphincteric resection (ISR) helps to preserve anal function. We hereby report our experience of evaluation and successful management in a rare case of rectal gastrointestinal stromal tumor (GIST), wherein we managed a case of rectal GIST with imatinib neoadjuvant followed by surgery and adjuvant therapy.

Keywords: Abdominoperineal resection, Gastrointestinal stromal tumor, Imantinib, Rectal tumor.

The Journal of Medical Sciences (2019): 10.5005/jp-journals-10045-00135
\end{abstract}

\section{INTRODUCTION}

Gastrointestinal stromal tumors (GISTs) are the most common mesenchymal tumor of GI (gastrointestinal) tract. It arises from the interstitial cells of cajal. ${ }^{1}$ It can arise from any site of the GI tract. The stomach is the most common site; however, it can arise from any site of $\mathrm{Gl}$ tract and rarely involves the anorectal region. ${ }^{2}$ The incidence of anorectal GIST is 0.018 per 100,000; this comprises about $2.8 \%$ of all GISTs. ${ }^{3}$ GIST at other locations had worse prognosis compared to that in the stomach. ${ }^{4}$ Surgery is the mainstay of treatment as it is resistant to conventional chemotherapy. However, tyrosine kinase inhibitors such as imatinib mesylate can be used in a neoadjuvant or adjuvant setting. ${ }^{5}$ Margin-free resection is the goal. Anatomical factors in the pelvic area lead to recurrence. There are few case reports and small series in the literature regarding the successful use of imatinib in the neoadjuvant setting followed by surgery. Here we are reporting a case of malignant GIST of lower rectum who was managed with abdominoperineal resection following neoadjuvant imatinib therapy and achieved a recurrencefree survival after 4 years of follow-up.

\section{Case Description}

Our patient was a 64-year-old male. He presented with complaints of bleeding in the rectum for the last 6 months. It was fresh blood alongside stool and was not associated with any postural symptoms; however, it was associated with tenesmus and occasional incontinence to flatus and stool. There was occasional colicky abdominal pain with distension which relieved after passage of flatus and stool. He had a loss of appetite and insignificant weight loss. There was no history of hematemesis, jaundice, respiratory distress, bony pain, or altered mental status. His bladder habit was normal. He had no comorbidities or any addictions. He had no cervical, axillary, or inguinal lymphadenopathy. Abdomen was soft, and there was no palpable lump or organomegaly. Digital rectal examination revealed a firm growth starting $1.5 \mathrm{~cm}$ from anal verge from the 4 to 8 o'clock position and the finger was not negotiable beyond the growth. The tumor was immobile and bleeding on touch. With the above history and clinical examination, diagnosis of a rectal growth was made. His hemogram, liver function test, renal function test, and coagulation parameters were within normal limits. Colonoscopy revealed an ulcerated growth at $3 \mathrm{~cm}$ from
${ }^{1-4}$ Department of Surgical Gastroenterology, Sanjay Gandhi Postgraduate Institute of Medical Sciences, Lucknow, Uttar Pradesh, India

Corresponding Author: Ashok Kumar, Department of Surgical Gastroenterology, Sanjay Gandhi Postgraduate Institute of Medical Sciences, Lucknow, Uttar Pradesh, India, Phone: +91 5222495537, e-mail: drashok97@gmail.com

How to cite this article: Kumar A, Ghosh N, Kumar A, et al. Neoadjuvant Imatinib Therapy Followed by Surgery in a Rare Case of Rectal Gastrointestinal Stromal Tumor. J Med Sci 2019;5(4):103-105.

Source of support: Nil

Conflict of interest: None

the anal verge and extending up to $7 \mathrm{~cm}$. The rest of the colon was normal. Biopsy revealed malignant GIST. It was positive for CD 117, CD 34, and S-100 with a mitotic activity of 35/50 HPF (high power field). Ki-67 index was 15\%. It was negative for vimentin, SMA, and desmin. Contrast enhanced CT and MRI pelvis showed an $8 \times 8.5 \times 8.1 \mathrm{~cm}$ heterogeneously enhancing exophytic mass lesion with central areas of necrosis in the distal one-third of the rectum and involving anorectal junction. There were few subcentimetric perirectal nodes (Figs 1 and 2). Adjacent structures e.g., prostate and seminal vesicles, were free. In view of sphincter involvement, neoadjuvant imatinib was prescribed for 6 months. The disease was stable according to the Choi response criteria. He was scheduled for surgery and underwent abdominoperineal resection. Intraoperatively, there was no signs of dissemination and the liver was normal. Intraoperative, hypogastric nerves and ureters were identified and preserved. There was a bulky tumor of about $1 \mathrm{~cm}$ from the anal verge involving posterior and right lateral wall. The coccyx was removed. There was no tumor perforation. The cut section showed a $9 \times 8 \mathrm{~cm}$ polypoidal mass with a distal margin of $1 \mathrm{~cm}$ and proximal margin $>5 \mathrm{~cm}$ (Fig. 3). The rest of the mucosa was normal. Histopathology revealed a $9.7 \times 7.2 \mathrm{~cm}$ growth and margins were free. Tumor was reactive for c-kit, DOG-1, and CD34. Ki 67 index was 20-30\%. It was negative for cytokeratin and desmin. Postoperatively, the stoma started functioning from day 3 and he tolerated enteral fluids and feeds well. On postoperative follow-up, no urinary or sexual dysfunctions were noted. He 


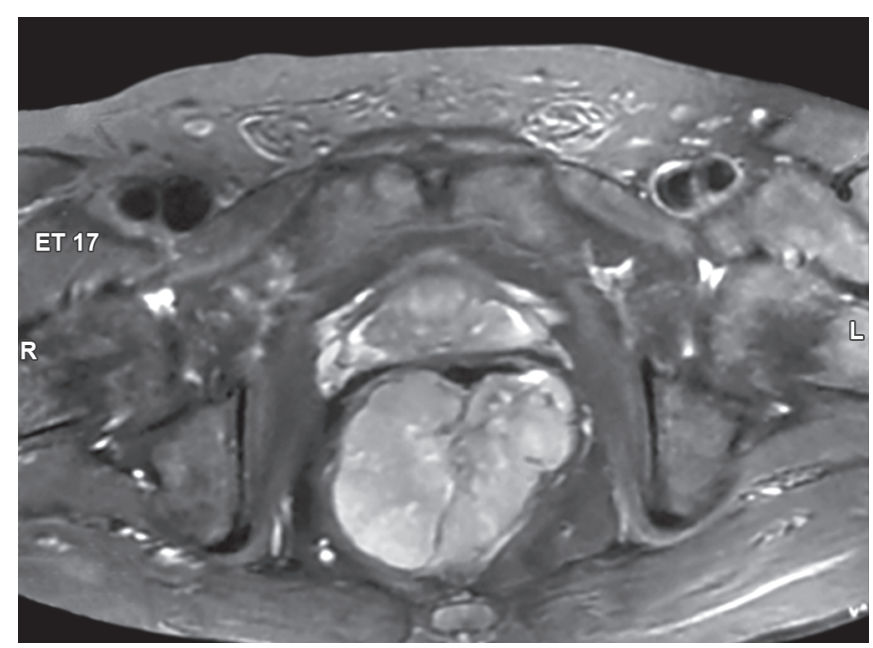

Fig. 1: CECT of the abdomen showing a large heterogeneously enhancing mass lesion arising from the posterior wall of the lower rectum with significantly narrowing lumen and indistinct fat planes

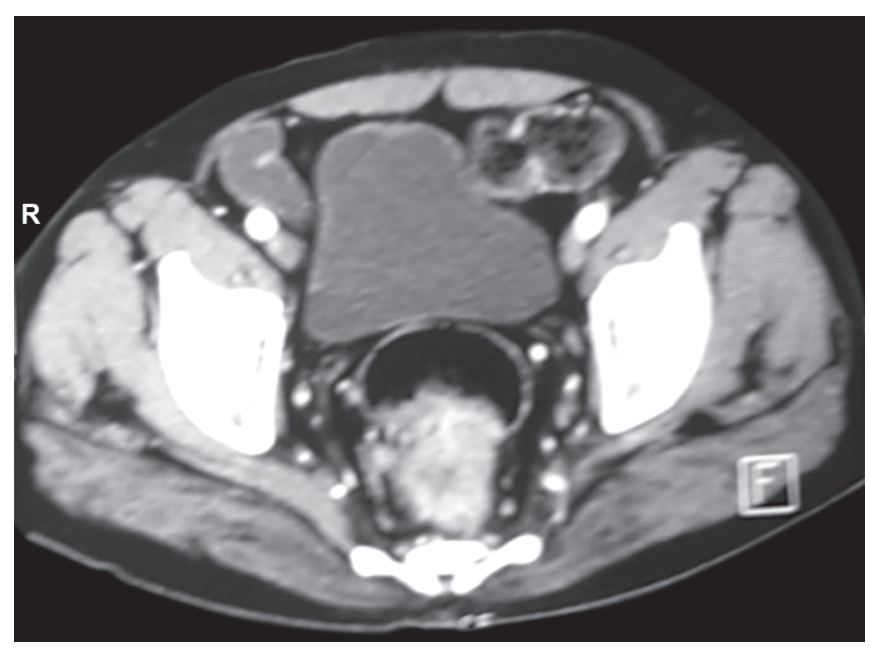

Fig. 2: MRI pelvis T2-weighed image showing a large heterogeneously enhancing mass lesion involving rectum and anorectal junction, central necrotic area within lesion, and fat with prostate are maintained was prescribed imatinib $400 \mathrm{mg}$ once a day for the next 3 years. After 4 years of follow-up, he is doing well and there was no evidence of recurrence of the disease.

\section{Discussion}

GIST is the most common mesenchymal tumor of the GI tract. The stomach is the most common site of involvement, followed by the small intestine. Although it can involve any part of the Gl tract, the rectum is rarely involved. Anorectal GIST is most commonly seen in males and in older aged population ( $>60$ years). The prognosis depends upon the site of involvement. ${ }^{6}$ Surgery with the goal of achieving R0 resection is the key. Standard antitumor chemotherapy and radiotherapy are ineffective. These tumors respond to tyrosine kinase inhibitors, i.e., imatinib, sunitinib, and regorafenib. These agents are used as adjuvants in the case of high-risk tumors for recurrence-free survival. ${ }^{7}$ These agents can be used in neoadjuvant setting to downstage the tumor and for sphincter preservation in anorectal GIST. There are various case series of rectal GIST operated after neoadjuvant imatinib in the literature, and in some of them

Table 1: Studies on rectal GIST

\begin{tabular}{|c|c|c|c|c|c|}
\hline Author & Year & No. of cases & Neoadjuvant & Surgery & Survival \\
\hline Sotiropoulos GC & 2017 & 1 & No & Local excision & No recurrence at 3 years \\
\hline Osama Eldamshety & 2017 & 2 & Yes ( 1 case) & Local excision (TAMIS) & No recurrence at 10 months \\
\hline Akiyoshi Takashi & 2014 & 3 & Yes & $\begin{array}{l}\text { Laparoscopic local excision with } \\
\text { rectoanal anastomosis }\end{array}$ & No recurrence at 3 months \\
\hline Fujimoto $Y$ & 2014 & 3 & Yes & Lap ISR & No recurrence at 4 years \\
\hline Sanjeev Singhal & 2013 & 1 & No & APR & - \\
\hline Kumar M & 2013 & 1 & No & APR & - \\
\hline Wang JP & 2011 & 3 & Yes & $\begin{array}{l}\text { Transanal excision and coloanal } \\
\text { anastomosis }\end{array}$ & No recurrence at 28 months follow-up \\
\hline Kyu Jong Yoon & 2011 & 2 & Yes & Ultralow AR & No recurrence at 6 months follow-up \\
\hline Machlenkin S & 2010 & 9 & Yes & Surgery & $\begin{array}{l}\text { Three patients had recurrence at median } \\
\text { follow-up of } 32 \text { months }\end{array}$ \\
\hline Garima Mehta $^{10}$ & 2010 & 1 & No & APR & - \\
\hline Ying-Yong Hou & 2009 & 1 & Yes & Segmental resection of colorectum & No recurrence at 57 months \\
\hline Yuma Ebihara & 2008 & 1 & Yes & APR & No recurrence at 24 months \\
\hline
\end{tabular}

ISR, intersphincter resection; APR, abdominoperineal resection; TAMIS, transanal minimal invasive surgery; Ultralow AR, ultralow anterior resection 
the sphincter could be preserved (Table 1). In our case, neoadjuvant imatinib, $400 \mathrm{mg}$ once a day, was prescribed for 6 months, and there was no significant improvement. Therefore abdominoperineal resection was done. There are few case reports from India, but imatinib was not used in the neoadjuvant setting..$^{8-10}$ This is the first case wherein neoadjuvant imatinib was used before surgery and a recurrence-free survival after 4 years could be achieved.

\section{Conclusion}

Imatinib can be used in the neoadjuvant setting of anorectal GIST to downstage the tumor. It could help to achieve sphincter preservation. Further, randomized large-scale studies are required to know its neoadjuvant and sphincter sparing action. This neoadjuvant therapy could be combined with other modalities of surgical resection of the tumor by using laparoscopy or robotics so that the anal function can be preserved.

\section{Clinical Significance}

Complete surgical resection with negative tumor margins is the key, and there is also a definite role of imatinib in the form of neoadjuvant and adjuvant therapy for better and disease-free survival.

\section{ACKNOWLedgment}

I wish to thank Prof VK Kapoor, professor and unit head of the Department of Surgical Gastroenterology, Sanjay Gandhi Postgraduate Institute of Medical Sciences, Lucknow, India, for guiding us and for his moral support and motivation.

\section{References}

1. Miettinen M, Lasota J. Gastrointestinal stromal tumors. Gastroenterol Clin North Am 2013;42(2):399-415. DOI: 10.1016/j.gtc.2013.01.001.

2. Tran T, Davila JA, El-Serag HB. The epidemiology of malignant gastrointestinal stromal tumors: an analysis of 1,458 cases from 1992 to 2000. Am J Gastroenterol 2005;100(1):162-168. DOI: 10.1111/j.15720241.2005.40709.x.

3. Liu Z, Wu S, Gou S, et al. A population-based study of the incidence and survival of anorectal gastrointestinal stromal tumor. Med Sci Monit 2019;25:5408-5417. DOI: 10.12659/MSM.915967.

4. Miettinen M, Lasota J. Gastrointestinal stromal tumors: review on morphology, molecular pathology, prognosis, and differential diagnosis. Arch Pathol Lab Med 2006;130(10):1466-1478.

5. Kaneko M, Nozawa H, Emoto S, et al. Neoadjuvant imatinib therapy followed by intersphincteric resection for low rectal gastrointestinal stromal tumors. Anticancer Res 2017;37(9):5155-5160.

6. Joensuu H. Risk stratification of patients diagnosed with gastrointestinal stromal tumor. Hum Pathol 2008;39(10):1411-1419. DOI: 10.1016/j.humpath.2008.06.025.

7. DeMatteo RP, Ballman KV, Antonescu CR, et al. Adjuvant imatinib mesylate after resection of localised, primary gastrointestinal stromal tumour: a randomised, double-blind, placebo-controlled trial. Lancet 2009;373(28):1097-1104. DOI: 10.1016/S0140-6736(09)60500-6.

8. Singhal $S$, Singhal A, Tugnait $R$, et al. Anorectal gastrointestinal stromal tumor: a case report and literature review. Case Rep Gastrointest Med 2013;2013:934875. DOI: 10.1155/2013/934875.

9. Kumar M, Goel MM, Singh D. Rare case of gastrointestinal stromal tumor of the anal canal. J Cancer Res Ther 2013;9(4):736-738. DOI: 10.4103/0973-1482.126476.

10. Mehta G, Verma S, Inamdar P. Anorectal leiomyoma: case report of rare tumor with unusual presentation. Indian J Surg Oncol 2010;1(4):337-340. DOI: 10.1007/s13193-010-0037-3. 